\title{
Documentos
}

\section{VULVODINIA: UN DIAGNÓSTICO OLVIDADO FRENTE AL DOLOR VULVAR}

\author{
Paolo Ricci A. ${ }^{1}$ \\ ${ }^{1}$ Unidad de Ginecología, Departamento de Obstetricia y Ginecología, Clínica Las Condes.
}

\section{RESUMEN}

La vulvodinia es una patología compleja y de difícil tratamiento. Se define como un dolor crónico de la vulva, que puede ser generalizado o localizado. La primera vez que se escribe sobre esta fue en 1889, por Skene. Sin embargo, es sólo hasta 1976, que los miembros de la International Society for Study of Vulvovaginal Diseases, reconocieron el dolor vulvar como una entidad patológica. Se estima una prevalencia de un 15\%, con unas 14 millones de mujeres que lo padecen en EEUU. Generalmente consultan múltiples veces y a diferentes médicos, antes de llegar a un diagnóstico. No existen pruebas específicas, por lo que la clínica y el examen físico son las principales herramientas. Se deben excluir todas aquellas patologías que explican este dolor crónico vulvar. Su etiología es multifactorial, involucrando cambios a nivel de nociceptores, alteraciones de la inervación y la presencia de factores inflamatorios. Sin embargo, no podemos dejar de lado los aspectos psicosexuales, que pueden modular o desencadenar el dolor vulvar, al encontrar un sustrato alterado a nivel de estos tejidos. Basándose en estos aspectos, hoy existen diferentes tratamientos, que son efectivos si los utilizamos asociados y en forma gradual, ya que se potencian entre ellos.

\section{PALABRAS CLAVE: Dolor vulvar, vulvodinia, disestesia vulvar, dolor vulvar crónico, vestibulitis vulvar}

\section{SUMMARY}

Vulvodynia is a complex pathology and difficult to treat. In 1889 Skene was the first who write about this. However, only until 1976 the members of the International Society for Study of Vulvovaginal Diseases, recognized the vulvar pain as a disease entity. Prevalence estimated at $15 \%$ with an estimated 14 million women who suffer in the USA. A high number of gynaecologists do not know this diagnosis. For this reason women consult multiple times and to different doctors to reach a diagnosis. There are no specific tests for diagnosis. Finding in the clinical and physical examination are the main tools for this diagnosis. The different pathologies that could explain the presence of a chronic vulvar pain must be excluded. Undoubtedly its aetiology is multifactorial, involving changes in the number of nociceptors and alterations in the innervations, and the presence of inflammatory factors that may be the starting point of this pathology, as it seeks to explain the neuropathic theory. However we can not ignore the psychosomatic theory in explaining the psychosexual disorders as a trigger vulvar pain, in these altered tissues. Based on these aspects there are now treatments that are more or less effective when are gradually associated for better results.

KEY WORDS: Vulvar pain, vulvodynia, vulvar dysesthesia, chronic vulvar pain, vulvar vestibulitis 


\section{INTRODUCCIÓN}

Los ginecólogos en su ejercicio profesional cotidiano, se enfrentan a una gran cantidad de patologías, que gracias a las nuevas investigaciones, se pueden comprender y tratar cada vez con mayor efectividad. Entre las posibles patologías a las que nos podemos enfrentar, se encuentran las que afectan a la vulva. Sin embargo, la vulva sigue siendo el órgano olvidado. Un ejemplo es el diagnóstico de la llamada vulvodinia, que es un desorden complejo que puede ser difícil de tratar (1). Es una patología que ha sido definida como un dolor de carácter crónico a nivel vulvar, sin una causa definida. Este dolor ha sido categorizado en el ICD-9, bajo el grupo 625, específicamente como 625,7 (dolor y otras alteraciones de los órganos genitales femeninos) (2). Durante el 2002 y nuevamente en el 2006 el NIH (National Institutes of Health) caracteriza la vulvodinia (V) como un síndrome doloroso focal pobremente entendido e investigado, para el cual el tratamiento óptimo es desconocido (3).

$\mathrm{La}$ experiencia de las mujeres que sufren V, suele ser pobremente comprendida por quienes las rodean e incluso por muchos de los médicos a los que consultan. Se trata de una patología que tiene efectos negativos sobre la calidad de vida (4).

Es un trastorno en el que hay un gran intervalo de tiempo perdido, entre la primera consulta por los síntomas y signos, y el momento en que alguien realiza el diagnóstico.

La ISSVD (International Society for Study of Vulvovaginal Diseases) ha definido a la vulvodinia como "malestar crónico vulvar, que se caracteriza por ardor, picazón, irritación o sensación de "carne viva", en ausencia de hallazgos visibles, clínicamente identificables, patología neurológica o infección de la piel" (5).

A pesar de las investigaciones desarrolladas para intentar descifrar su etiopatogenia, especialmente en las últimas dos décadas, la causa no se conoce completamente. Mientras que un tratamiento efectivo, sigue siendo difícil.

\section{HISTORIA}

Esta entidad fue descrita por primera vez en 1889, por Skene, como una "excesiva sensibilidad vulvar" (6). En 1891 Thomas y Munde plantearon que se debía a una excesiva sensibilidad de las terminaciones nerviosas de la mucosa vulvar (7). Kelly en 1928 describió casos de dispareunia debido a dolor frente a la estimulación de zonas rojas y sensibles en la mucosa del introito (8). Luego hay un período de 5 décadas en que no se hace referencia en la literatura médica a esta patología. En 1976, los miembros de la ISSVD reconocieron el dolor vulvar, como un entidad única y la llamaron síndrome de ardor vulvar (burning vulvar syndrome). Sin embargo, sólo en 1983, la ISSVD utiliza el término de vulvodinia. En 1985, la clasifican en dos tipos, la disestesia vulvar y la vestibulitis, según el lugar que afecta (9). En 1999, se utiliza el término vestibulodinia. Durante el 2003, nuevamente se revisa el término, y la ISSVD decide usar genéricamente el nombre de vulvodinia, que utilizamos hasta hoy. La clasifica en generalizada o localizada, y según la presencia o ausencia de estímulos para dolor (10) (Tabla I-II).

\section{PREVALENCIA}

Un estudio de 2127 mujeres determinó que en los últimos 6 meses un 3,8\% de las mujeres encuestadas había presentado $\mathrm{V}$, con una prevalencia a lo largo de la vida de 9,9\% (11). Dos estudios

\section{Tabla I}

\section{CLASIFICACIÓN DE LA VULVODINIA (ISSVD)}

A. Localizada (vestibulodinia, clitorodinia) provocada (por estímulos dolorosos sexuales, no sexuales o ambos) espontánea (ausencia de estímulos dolorosos) mixta

B. Generalizada provocada (por estímulos dolorosos sexuales, no sexuales o ambos) espontánea (ausencia de estímulos dolorosos) mixta

ISSVD: Society for Study of Vulvovaginal Diseases

Tabla II

\section{TÉRMINOS UTILIZADOS EN LA EVOLUCIÓN HISTÓRICA DE LA VULVODINIA}

Términos antiguos:
vulvodinia esencial
disestesia vulvar
vulvovaginitis psicosomática
vulvodinia disestésica
síndrome de vestibulitis vulvar

Término actual:

vulvodinia generalizada

vulvodinia localizada 
publicados durante el 2008, determinaron que durante un año, se presentará 1 caso nuevo cada 20 a 50 mujeres. Mientras que a lo largo de un año, sólo 1 de cada 10 mujeres que padecen $\mathrm{V}$, tendrá remisión de los síntomas (12). Otras revisiones relatan que la $V$ es la causa de deterioro en la salud mental, física y reproductiva, entre el $4 \%$ (13) y el $16 \%$ de las mujeres, en algún momento de sus vidas (4). Esto permite estimar que al menos 14 millones de mujeres en EEUU, pueden padecer de $V(4,13)$, y sólo dos tercios consultarán un médico por esta razón (14). Un $15 \%$ de las mujeres que consultan al ginecólogo presentan $V(15)$. Afecta preferentemente a mujeres adultas, sin embargo también pueden padecer de $\mathrm{V}$ las niñas pre-adolescentes (16). Respecto a la distribución racial/étnica, una revisión en 26 mujeres en un centro médico universitario de Texas, demostró una distribución de: $85 \%$ hispánicos, $11 \%$ afro-americanos y $4 \%$ caucásicos (17).

\section{FISIOPATOLOGÍA}

La causa de la $V$ es desconocida, hoy sabemos más sobre lo que no es su causa. Sin embargo, desconocemos en su totalidad los factores que la desencadenan o facilitan. La $\mathrm{V}$ no es causada por las infecciones comunes de la vagina y vulva, como la cándida Sp., papilomavirus humano, herpes, etc. No es causada por patología inflamatoria, como el liquen plano o alteraciones inmunológicas. No es causada por neoplasias, como el Paget o el carcinoma de células escamosas. No es causada por alteraciones neurológicas, como la neuralgia herpética y compresión de nervios a nivel espinal (1).

La vulva sigue siendo el "órgano olvidado". Un estudio ultraestructural del epitelio en las mujeres con patología vulvar, nos podría dar muchas respuestas que orienten un tratamiento adecuado. Sin embargo, esto requiere un conocimiento detallado de la superficie epitelial de la vulva normal (1).

Se ha propuesto que puede ser causa de anormalidades embriológicas. Un rol importante se ha atribuido a las dietas ricas en oxalatos, factores genéticos, inflamatorios, infecciosos y cambios neuropáticos. En base a la experiencia de los pacientes tratados, sólo podemos decir que lo más probable es que sea el resultado de la suma de diferentes factores (1).

Para intentar comprender la complejidad de esta patología y la falta de conocimientos mayores sobre su etiología, debemos mencionar que la región vulvovaginal está formada por epitelios que tienen origen en las tres capas embriológica (ectodermo, mesodermo y endodermo). Esto constituye una condición especial no vista en otra parte del cuerpo. Quizás lo más parecido es la cavidad oral. Cada una de estas tres derivaciones embriológicas es diferente en cuanto a las estructuras epiteliales y glandulares, respuesta hormonal, distribución neurológica, respuesta inmune, y en su relación y comportamiento frente a patologías que afectan otros sistemas (18).

La región vulvo-vaginal es la zona anatómica que permanece más tiempo cubierta por la ropa en el cuerpo. Por lo que puede verse afectada por contacto o roce, por las prendas íntimas, que pueden estar formadas por tejidos o telas, que a veces contienen sustancias acrílicas, artificiales, que causan humedad y estímulo irritativo permanente, e incluso maceración de tejidos. Por medio de inmunohistoquímica se ha demostrado alteración de la densidad de terminaciones nerviosas y receptores de estrógeno (19).

Frente a los conocimientos entregados por algunas investigaciones, es probable que exista un rol en la génesis de la $V$, a través de defectos en la regulación inmunológica, llevando a una condición pro-inflamatoria (20). Una elevada concentración de citoquinas inflamatorias, ha sido demostrada en mujeres con V, al compararlas con controles asintomáticas. Estas son interleukina 1 beta, IL-6, IL-8 y el factor de necrosis tumoral alfa, y podrían contribuir en la fisiopatología de la hiperalgesia mucocutánea (21).

Un estudio realizado por Foster y cols (22), demostraron que existe una condición genética, que aumenta la probabilidad de desarrollo de $\mathrm{V}$ localizada en el vestíbulo. Esta variante genética, provocaría cambios a nivel de IL1RN (receptor antagonista de interleukina 1) y MC1R (receptor de melanocortina 1), cuya combinación se asocia con un riesgo mayor.

Diferentes estudios han demostrado una hiperplasia de los nervios en la mucosa de mujeres que padecen vestivulitis vulvar (23). Esto podría ser otro factor en la etiología de este dolor crónico, que se suma o se presenta independiente de los mediadores inflamatorios. Mediante inmunohistoquímica se han estudiado estas terminaciones nerviosas, identificando neuropéptidos propios de nociceptores (24). Como el "péptido relacionado con el gen de la calcitonina", que además es un poderoso dilatador de arteriolas (24). Se ha encontrado también un aumento del receptor TRPV1, que se sabe es estimulado por la capsaicina, protones, calor nocivo y productos producidos por inflamación (25). Estos receptores han sido identificados, en aumento en otras patologías que se presentan con hiperalgesia, en diferentes lugares del cuerpo humano (26). Esto 
último permite unir ambas teorías, la de aumento de terminaciones nerviosas específicas y la presencia de un factor de inflamación. Entonces surge la pregunta sobre qué es primero, ¿la inflamación o los cambios en los nociceptores?. Algunos sugieren que un proceso inflamatorio podría iniciar cambios neurofisiológicos, incluyendo la proliferación y la sensibilización de los nociceptores $(24,27)$, y un aumento progresivo de la actividad de las neuronas medulares a nivel del asta dorsal, por una permanente estimulación de las fibras aferentes del tipo C (28). Otras investigaciones apoyan la teoría que también aumenta la inervación y/o sensibilización de termo-receptores (27).

Si pensamos en una inflamación crónica como punto de partida, esto le da una especial importancia a la observación, que muchas de estas pacientes, tienen múltiples consultas previas, debido a vulvovaginitis crónica o procesos infecciosos a repetición. Este proceso de irritación crónica, a través de las sustancias ya descritas, llevaría al aumento exagerado de vasos sanguíneos y vasodilatación, lo cual provocaría cambios en la mucosa y el eritema característico. Sin embargo, la intensidad del eritema no va en relación directa a un aumento mayor de la perfusión, debido a la vasodilatación y formación de neovasos. Al parecer se debería a características propias de la mucosa de cada paciente y otros factores (29).

Por otro lado, si el punto de partida es local y específicamente en la alteración de receptores o terminaciones nerviosas y la producción de sustancias pro-inflamatorias, permite suponer que terapias tópicas que actúan antagonizando a estos receptores, podrían ser un tratamiento exitoso a futuro.

Es frecuente que estas mujeres con antecedente de vulvovaginitis crónica, queden sin un estudio adecuado, recibiendo múltiples terapias tópicas, fundadas en una observación teórica de lo que se cree o parece ser una micosis. Ashman y cols (30), desarrollaron una teoría en la que antígenos de la cándida albicans, a través de reacción cruzada, llevarían a la producción de auto-anticuerpos que actuarían secundariamente contra la mucosa vulvovaginal. Por otro lado, el antígeno que se ha aislado es de $48 \mathrm{kDa}$ y muestra una homología con la enzima enolasa (31). Incluso, Marinoff y Turner (32), creen que esta hiperreactividad podría desarrollarse a partir del uso frecuente e indiscriminado de antimicóticos tópicos.

Llama la atención lo encontrado por estudios con capsaicina, que es utilizada para evaluar la sensibilización central, en la investigación del dolor.
Las mujeres con $\mathrm{V}$ presentaron mayor dolor frente a un estímulo, además de una frecuencia de pulso en reposo mayor, y una presión arterial sistólica más baja que los controles. Es decir hay una respuesta diferente frente al dolor, que se extiende posiblemente al resto del cuerpo, y a un funcionamiento central diferente (33).

Otros trabajos que apoyan un rol central en la $\mathrm{V}$, son aquellos que demuestran un aumento de la sensibilidad y dolor frente a la presión, tanto en la vulva, como en zonas periféricas del cuerpo. Si esto juega un rol mayor en la etiología de la $V$ en algunas mujeres, podría permitirnos comprender porqué algunas terapias específicas en este sentido tienen mayor efecto, como es el caso de los antidepresivos, la terapia cognitivo-conductual y los ejercicios aeróbicos (34). Pukall y cols (35), demostraron que estas mujeres tenían mayor sensibilidad en el deltoides también.

Por otro lado, la intensificación de la percepción de los estímulos nocivos en estas mujeres, puede sugerir la alteración de mecanismos neuroquímicos en la experiencia del dolor, que pueden estar afectados y modulados por el tipo de personalidad, lo cual nos permite sugerir que también habría factores psicológicos involucrados (36).

No puedo dejar de mencionar que hay trabajos que han demostrado un aumento de la presión uretral y de su tono muscular (37). Sin embargo, no podemos dejar de lado el vaginismo, que frecuentemente se puede asociar y ser la causa del aumento del tono muscular del suelo pelviano en general.

Durante el año 1996 y 2000, estudios demostraron que podría haber un soporte en la idea que el virus papiloma humano, podría tener alguna relación en la etiopatogenia de la $V$. Esta relación fue débil, pero la prevalencia del HPV fue el doble que los controles (50\% vs $23,9 \%)(38,39)$. Sin embargo, la mayoría de los trabajos demuestra que no existe tal asociación, y que la existencia de HPV es sólo incidental, no existiendo una real relación causal (40). Incluso en el pasado se llegó a utilizar el término vulvitis por papilomavirus humano, lo que más tarde se planteó como un error, por no existir una relación causal (41).

Después de analizar estos trabajos que contribuyen a intentar comprender algo más de este dolor crónico, podemos decir que hay tres sistemas interdependientes involucrados: la mucosa vulvar, los músculos pelvianos y las vías neurológicas. Sin lugar a dudas la vulvodinia debe ser el resultado de procesos multifactoriales, que involucran a estos tres sistemas (42). 


\section{COMORBILIDAD, DESENCADENANTES Y VUL- VODINIA}

Nguyen y cols (43), han demostrado que diversos episodios de infecciones urinarias pueden preceder a la aparición de vulvodinia. También se ha correlacionado con una historia crónica de episodios de vaginitis (4). En un estudio de casos y controles, fue mayor el riesgo en quienes tenía historia de vaginosis bacteriana $(O R=9,4)$, candida albicans $(O R=5,7)$, enfermedad inflamatoria pélvica $(O R=11,2)$, tricomoniasis $(O R=20,6)$, y displasia vulvar $(\mathrm{OR}=15,7)$. No se asoció riesgo de padecer $\mathrm{V}$ al antecedente de infección por VPH (virus papiloma humano), ASCUS, displasia cervical, clamidias, verrugas genitales, herpes genital o gonorrea (44).

En la literatura se describen casos de dolor vulvar crónico, después de producirse una infección herpética. Este antecedente podría hacernos pensar en una neuralgia posherpética, en que se produce una irritación e inflamación de algunos nervios, lo que producirá un dolor según la zona afectada. El herpes vulvar corresponde a una presentación poco común, de una enfermedad común. Se estima que en EEUU, 1,5 millones de mujeres lo padecen a lo largo de su vida, y 150.000 de ellas presentan una neuralgia posherpética (45). Se estima que un $8,4 \%$ de los herpes pueden afectar los dermátomos que inervan la vulva (46). Sin embargo, una diferencia con la vulvodinia, es que las neuralgias posherpéticas afectan de preferencia a mujeres de mayor edad.

La relación de la presencia de cistitis interticial y/o dolor vesical, con $V$ ha sido establecida en varias revisiones (47). Actualmente se dice que esta asociación está subestimada, reportándose cifras entre el 25 a $50 \%$ de los casos. En una revisión de 197 mujeres con estas patologías, se encontró que el $10,9 \%$ presentó dolor vulvar durante la adolescencia, mientras que el $48,4 \%$ lo presentaba en la etapa de adulto (48). En nuestra experiencia, encontramos esta asociación en el $25 \%$ de los casos que tenemos en tratamiento.

Es bastante frecuente encontrar la $V$ asociada a otras comorbilidades. Las revisiones han demostrado que en más del $50 \%$ de las mujeres que padecen V, hay asociación a patologías crónicas. Estas mujeres utilizan más sus sistemas de salud que los casos controles. Diferentes estudios demuestran que entre las más frecuentes, se encuentra el síndrome de fatiga crónica (13\%), fibromialgia $(20 \%)$, migraña-cefalea (31\%), colon irritable $(35 \%)$, otros dolores crónicos (41\%) y dolor lumbar (56\%) $(4,49)$.

La dispareunia (41) y el vaginismo (50) son dos problemas, que podemos ver secundarios a la mantención en el tiempo de la $\mathrm{V}$ (51). El vaginismo se puede presentar como una "respuesta de protección" frente al dolor. Hacer un diagnóstico diferencial se hace difícil cuando identificamos signos y síntomas de estas patologías asociadas. Existen revisiones que muestran que esta asociación podría ser alta (52). En nuestra experiencia se da en el $70 \%$. En la literatura médica se ha encontrado mayor frecuencia de síndrome premenstrual y dismenorrea en quienes padecen $\mathrm{V}$ (49).

Un estudio buscó la presencia de vulvodinia en mujeres portadoras de VIH. Para ello se compararon 235 mujeres positivas versus 272 controles negativas, encontrando 5 casos de $V$ en las primeras y sólo 1 en las otras. Sin embargo, se requieren más investigaciones para poder decir algo sobre esta asociación (53).

\section{CLÍNICA}

El diagnóstico de esta patología se basa en la exclusión de otras etiologías (Tabla III). Se debe descartar el dolor de una causa identificable, como vulvovaginitis por cándida a repetición, liquen plano, herpes genital, etc.

\section{Tabla III}

\section{DIAGNÓSTICO DIFERENCIAL DE VULVODINIA}

Diagnóstico $\quad \begin{gathered}\text { Presentación clínica } \\ \text { más frecuente }\end{gathered}$

Herpes vulvar

Vulvitis alérgica

Atrofia vulvar

Liquen plano

Liquen escleroso

Síndrome pudendo

Neoplasia

intraepitelial vulvar

Endometriosis

vulvo-vaginal
Lesiones vesiculares dolorosas

Prurito, ardor, irritación

Piel y mucosa pálida y delgada

Lesiones reticuladas blancas, prurito, ardor

Pueden coexistir lesiones en boca

Lesiones blancas, piel adelgazada y arrugada Prurito que puede ser severo

Dolor unilateral generalmente y que aumenta al sentarse

Lesiones blancas 0 multicolores,solevantadas, muchas veces de aspecto verrucoso

Asintomáticas o pruriginosas

Lesiones blandas de lento crecimiento, que duelen con la menstruación 
Su característica es el dolor de la vulva, que lo describen como "de carne viva", quemante y/o escozor. La mayoría (88\%) dice que es sensación de quemadura. El dolor puede ser intermitente o constante, y debe estar presente en los últimos 6 meses o más (11).

La $\mathrm{V}$ se clasifica actualmente según su localización. Puede ser generalizada o localizada (Tabla I). Esta última puede afectar al vestíbulo vulvar, recibiendo la denominación de vestibulitis vulvar (5). Este dolor al ser localizado puede afectar zonas específicas como el clítoris (clitorodinia) (3). En ambos tipos de $\mathrm{V}$, tanto la generalizada como la localizada, el dolor puede ser espontáneo o provocado por estímulos, como la presión de una pequeña torunda de algodón. También puede gatillarse por contacto físico sexual. Sin embargo, estas características, que parecen muy fáciles de distinguir, se pueden superponer. Existiendo zonas de dolor provocado frente a estímulos y espontáneos, es decir de características mixtas. El éxito de los diferentes tratamientos se asocia a un mejor o menor resultado de acuerdo al subtipo de dolor vulvar. Inmediatamente surge la pregunta sobre si la vulvodinia generalizada y la localizada son el resultado de diferentes etiologías. Sin embargo, a través de los estudios de pacientes, se ha determinado que las características de ambos grupos de mujeres son similares, apoyando más la teoría que ambos subtipos del trastorno pueden incluso presentarse como un continuo de severidad y no corresponden a entidades diferentes (54). Sin embargo, la presentación del tipo localizado es más frecuente que la generalizada. Además, el dolor que puede ser localizado o generalizado, se puede denominar según si se presenta a partir del primer coito o utilización de tampón (primario), o si aparece después (secundario) (55).

La mayoría (57\%) de los casos presentan los síntomas después de los 30 años. En un quinto de las mujeres se presenta antes de los 20 años. El $65 \%$ ha consultado entre 3 y 9 médicos por sus síntomas (56).

\section{DIAGNÓSTICO DIFERENCIAL}

El dolor vulvar ha sido clasificado en aquel de causa conocida, es decir secundario, que puede deberse a una infección o patología dermatológica de la piel y mucosa genital. Sin embargo, esto no corresponde a la vulvodinia o dolor vulvar crónico esencial primario, que es el tema que nos encontramos analizando.

Entre los diagnósticos diferenciales (Tabla III) está la vulvitis alérgica, que puede presentarse con cambios en la piel y mucosa vulvar, como eritema, además de prurito y/o ardor. El liquen escleroso puede dar un prurito severo, generalmente se observan lesiones blancas y adelgazamiento de la piel. El liquen plano se identifica con lesiones blancas reticuladas, prurito y ardor; puede asociarse a erosión, eritema, lesiones bucales o placas papuloescamosas. La atrofia vulvar se puede presentar en mujeres con hipoestrogenismo, por lo que generalmente se presenta durante la postmenopausia; se observa la piel pálida, adelgazada y arrugada. El síndrome del canal pudendo, es generalmente unilateral, y suele aumentar al sentarse (57). La neoplasia intraepitelial vulvar se presenta como lesiones blancas o de múltiples colores, con solevantamiento, de aspecto verrucoso; pueden ser asintomáticas o pruriginosas. Aunque muy poco frecuente, la presentación de endometriomas extrapélvicos es posible; al encontrarse en vulva y vagina, se observan como lesiones blandas de crecimiento lento.

Aún cuando el vaginismo se puede asociar a $\mathrm{V}$, es necesario diferenciarlo (50). Igualmente puede agregarse dispareunia, produciendo dolor durante el coito. De esta forma, se puede realizar un tratamiento ajustado a una vulvodinia que se presenta sola o aquella asociada a vaginismo y/o dispareunia.

\section{DIAGNÓSTICO}

Los síntomas son la principal base del diagnóstico. Sin embargo, lo más importante es descartar una posible causa identificable del dolor. Ya que si se encuentra una patología que lo provoque, el tratamiento de esta llevará a la desaparición del dolor. Con la historia clínica compatible, al examinar en la consulta médica, se realiza el $96 \%$ de los diagnósticos (58).

Para identificar las zonas dolorosas, se puede utilizar la compresión por medio de una pequeña mota de algodón o torunda. Existen otros test para identificar y estudiar el dolor, uno es la prueba del tampón. Generalmente tienen dolor al colocarse tampones (59), sin embargo, no debemos guiar el diagnóstico por este dato. Ya que muchas de ella no lo han utilizado nunca. Debemos preguntar este dato en la historia clínica, ya que al existir podría ser de ayuda, muchas de ellas lo expresan como el primer síntoma.

Es muy frecuente observar zonas de eritema en la vulvodinia generalizada y en la vestibulitis vulvar. Este cambio de coloración de la mucosa puede ser en diferentes grados, tal como fue establecido por los criterios de Friedrich, en 1987 (60) (Tabla IV). 
Estas zonas generalmente corresponden a áreas de dolor. Una investigación demostró, por medio de mapeo a través de doppler láser, un aumento del flujo sanguíneo superficial, especialmente en la mucosa vestibular posterior. Que según nuestra experiencia, en un alto porcentaje se encuentra comprometida con eritema, cuando el diagnóstico es vestibulitis vulvar. Es muy probable que el aumento de la perfusión esté causado por una vasodilatación neurogénica.

\section{Tabla IV \\ CRITERIOS DESCRITOS POR FRIEDRICH, PARA EL DIAGNÓSTICO DE VESTIBULITIS VULVAR}

Dolor severo en el vestíbulo vulvar al contacto o intento de entrada en la vagina

Sensibilidad a la presión localizada en el vestíbulo vulvar

Eritema vulvar (inflamación) de diversos grados

En la vestibulitis vulvar el dolor está localizado preferentemente en la mucosa alrededor del ducto de salida de la glándula de Bartholino, región vestibular posterior, incluyendo el área del himen (61).

La biopsia de las zonas dolorosas generalmente muestra inflamación crónica en los tejidos subepiteliales, frecuentemente alrededor de los ductos de las glándulas vestibulares menores, lo que no constituye una característica específica, que permita la diferenciación de otras patologías (61).

\section{TRATAMIENTO}

Como todos los síndromes dolorosos, que teóricamente en su génesis tienen un componente producido por mediadores inflamatorios, y que en muchos de ellos desconocemos. Sólo una vez que descifremos cuáles de estos mediadores están aumentados, podremos actuar directamente sobre ellos, con una respuesta efectiva. Es posible que ahí esté la llave al éxito en el tratamiento de todos los síndromes dolorosos. Mientras tanto el estándar en estos síndromes es la inhibición o supresión de mediadores inflamatorios y la inhibición, supresión o modulación de los aferentes neuronales y la transmisión eferentes (motores) (62). Aunque las ciencias básicas y la investigación clínica ya han dado algunas interesantes observaciones, aún es más lo que desconocemos, por lo que muchos tratamientos se basan en la teoría y otros en la observación y medicina de la evidencia.

La terapia debe ser secuencial, y contempla desde tratamientos con cremas tópicas, antidepre- sivos, dieta baja en oxalatos, pasando por la kinesiterapia, psicoterapia, hasta la cirugía.

Una revisión de 38 trabajos sobre tratamientos en vulvodinia, determinó que sólo 5 correspondía a ensayos randomizados. Por otro lado, la mayoría tiene defectos metodológicos, como: ausencia de grupos controles o con placebo, evaluación doble ciego, evaluación del dolor antes del tratamiento, uso de medidas validadas de dolor y de las funciones sexuales. Sin embargo, los estudios existentes demuestran que los resultados con los tratamiento expuestos en este manuscrito, son alentadores. No debemos olvidar que el tratamiento óptimo es aún desconocido (3).

Medidas generales: Se debe disminuir todo lo que signifique o pueda irritar la zona vulvar. La ropa interior debe ser de algodón, evitando otras telas. No utilizar perfumes en la zona vulvo-perineal. No utilizar jabones que irriten, preferentemente agua sola para el lavado. Lavar muy bien la ropa interior, evitando que queden productos detergentes y suavizantes. No utilizar ropa interior para dormir. No utilizar toallas higiénicas que no sean de algodón. Evitar el uso de panty o pantalones ajustados en la zona de los genitales. Evitar el excesivo lavado en la zona vulvar.

Medicamentos: Se han utilizado exitosamente los antidepresivos tricíclicos, lográndose buen control del dolor, que puede llegar hasta el 60\% de los casos (63). También se han indicado anticonvulsivantes, tanto vía oral como tópica $(3,64)$. Los anestésicos tópicos de liberación sostenida es otra opción (65). Sin embargo, se deben tener presentes los efectos adversos, especialmente los anticolinérgicos de los antidepresivos tricíclicos. La sedación y los mareos son frecuentes en el uso de anticonvulsivantes, lo que puede interferir en el tratamiento. Aunque escasas, hay investigaciones que evalúan el uso de doxepina (66), cromolina (67), nitroglicerina (68) y capsaicina (69). También se utilizan infiltraciones con corticoides $(70,71)$. Se necesitan estudios controlados, doble ciego, para definir si son terapias beneficiosas en el control del dolor de estas mujeres.

Fisioterapia: Son múltiples los estudios que han demostrado que la fisioterapia es eficaz en la disminución y control del dolor (51). La electro-estimulación con "biofeedback" de la musculatura del suelo pelviano, es un tratamiento efectivo para la V (72). El biofeedback permite lograr una relajación voluntaria y aprendida de la musculatura. Esto es más importante aún, cuando se encuentra asociado vaginismo, como producto de la hipertonicidad muscular y los factores psicosociales y psicosexuales involucrados. Además, la electroanalgesia es segura y efectiva sobre el dolor, 
lo que en la mayoría de los casos se traduce en una mejoría en la dispareunia secundaria (73). La estimulación transcutánea (TENS) también ha demostrado ser efectiva en el control del dolor (74). Es frecuente encontrar diferentes grados de hipertonicidad muscular, por lo que la kinesiterapia es efectiva (75).

Acupuntura: Ha sido utilizada en el control del dolor en múltiples síndromes crónico. Una publicación realizada durante 1999, en que se utilizó semanalmente en doce pacientes, en sólo 3 mujeres no se vio efecto alguno. En 7 disminuyó la intensidad, sintiéndose mejor, y sólo 2 quedaron definitivamente sin dolor (76). Estos buenos resultados, aunque son en un grupo reducido, impulsa a repetir la experiencia en grandes series, para poder definir el beneficio de esta terapia como complemento a las otras medidas.

Hipnoterapia: Existe un estudio que demuestra que podría ser útil en la reducción del dolor y la restauración de las relaciones sexuales (77). Aunque es probable que su mecanismo sea directamente por lograr una mayor relajación, que podría actuar directamente en un posible vaginismo agregado, como consecuencia natural de la permanencia del dolor.

Cirugía: La vestibulectomía es un tratamiento efectivo para la vestibulitis vulvar (51). Puede ser muy controversial cuando planteamos una cirugía como tratamiento de un dolor "idiopático", sin embargo, muchos estudios demuestran su eficacia. Desde luego, lo ideal es dejar esta opción de tratamiento como una de las últimas. Utilizándola cuando se ha registrado falla de las otras terapias no quirúrgicas (78). Respecto a los resultados de la cirugía, algunas publicaciones sugieren que serían mejor frente a un dolor bien localizado y provocado. Se han utilizado diferentes técnicas para retirar la zona con inflamación y realizar una resección de la mucosa lo más reducida posible. Entre estas técnicas se describe la realizada por láser de contacto Yag (79). Los buenos resultados de la cirugía como terapia, confirman la teoría de los nociceptores en la fisiopatogenia de esta enfermedad (27). Algunos trabajos muestran un éxito completo con cirugía, de hasta el $57 \%$ de los casos, y disminución de los síntomas en un $89 \%$ (80). Un $68 \%$ dice sentirse curado de la dispareunia y $24 \%$ la disminuyó (81). Además estos buenos resultados se mantienen en el tiempo y aumentan más aún durante el primer año después de la cirugía (82). Sin embargo hay que tener en cuenta los costos, riesgos, y posible formación de nuevas cicatrices que pueden agregar un problema o constituir el punto de partida para la génesis de un nuevo dolor.
Dieta: Diferentes publicaciones sugieren que una dieta con restricción de oxalatos, podría ser beneficiosa. Esto se puede complementar con aporte de citrato de calcio, lo que podría ser más efectivo aún (83). Sin embargo, una experiencia con 7 mujeres en las que se diagnosticó hiperoxaluria (oxalatos en orina $>50 \mathrm{mg} / 24$ horas), se restringió la dieta (baja en oxalatos) y se agregó citrato de calcio, logrando una mejoría objetiva sólo en una (72). En otra serie se logró una mejoría del $50 \%$ de las mujeres que recibieron citrato de calcio (84). Otras revisiones en que se ha constatado la presencia de una dieta rica en oxalatos, en pacientes en que no se ha demostrado excreción urinaria anormal, se ha restringido igualmente en la dieta, logrando buenos resultados. Llegando a respuesta beneficiosa hasta en el $37 \%$ de los casos (85). Estudios que revisan la dieta de quienes padecen $\mathrm{V}$, no demuestran diferencias con casos controles. Los oxalatos urinarios pueden constituir un irritantes inespecífico que agrava una vulvodinia, sin embargo, su rol como iniciador de esta patología es poco probable.

Psicoterapia: La terapia cognitivo-conductual ha demostrado ser eficaz al utilizarla asociada en estas pacientes (86). Las investigaciones sugieren que las mujeres con $\mathrm{V}$, se benefician con el tratamiento que considera la focalización del dolor y sus componentes psicosociales (87). Muchas veces esta situación deteriora la relación de pareja, por lo que debe considerarse al varón en la psicoterapia (88). Los resultados de diferentes trabajos dan un soporte a la conceptualización psicosocial del dolor en la vulvodinia. Por otro lado, al ser frecuente la asociación de vaginismo, que podría interpretarse como una respuesta de protección frente al dolor experimentado durante las relaciones sexuales, necesariamente involucrará a la pareja, por lo que la psicoterapia realizada por especialistas en sexualidad, tiene una especial importancia. Por estos motivos, también se debe incorporar a la pareja.

El tratamiento de la $V$ debe ser integral y considerar la participación multidisciplinaria. Es así como son muy importantes la participación de la kinesiterapia y psicoterapia.

En los últimos años se han presentado algunas investigaciones con nuevas aproximaciones en el tratamiento de mujeres que no responden a las terapias convencionalmente presentadas. Uno de estos es la estimulación cortical transcraneal, no invasiva (89). Se trata de un método de neuromodulación, que ha sido utilizada en otros dolores crónicos, suponiendo una etiología o al menos una participación del nivel central. 


\section{PRONÓSTICO}

Mientras más tiempo pase sin tratamiento una mujer que padece de $\mathrm{V}$, más difícil será su tratamiento y menores sus resultados. Una de las razones es que con el tiempo se agregan otras morbilidades relacionadas, tal como se ha descrito en los puntos anteriores. Por otro lado es más factible que se desarrollen problemas de tipo psicológicos. Hay que tener presente que los tratamientos son de largo tiempo, por lo que no podemos esperar resultados inmediatos.

Algunos estudios han demostrado que la característica del dolor, si es primario o secundario, puede constituir un factor predictivo de los resultados de los tratamientos. Sin embargo, otros no logran demostrar esta asociación.

Mientras mayor es la intensidad del dolor antes de iniciar el tratamiento, puede ser un predictor de menores resultados para kinesiterapia con biofeedback, psicoterapia cognitivo-conductual y para cirugía. La presencia de erotofobia predice un pobre resultado frente a la vestibulectomía (90). En general los resultados se relacionan con la severidad del dolor y los factores psicosexuales (nivel de evidencia II).

En general, la mayoría de los estudios muestran una mejoría completa o cura en el $70 \%$ de las mujeres. Sin embargo, lo importante es que las mujeres que padecen $\mathrm{V}$, deben saber que con los tratamientos al menos se logra disminuir los síntomas, logrando retornar a la vida lo más normal que se pueda. Además hay que advertirles que la mejoría es producto de un proceso terapéutico muy lento y gradual.

Lo ideal es un mínimo de 2 a 3 meses de prueba con cualquiera de las terapias medicamentosas, con un aumento gradual, antes de evaluar un cambio. Una vez que se logra mejoría, no se debiera disminuir el tratamiento antes de 6 meses. Lo importante es expresarles a quienes padecen de $\mathrm{V}$, que el dolor que tienen es real, y no es producto de su imaginación, que no es el resultado de una enfermedad de transmisión sexual, ni tampoco un cáncer.

\section{CALIDAD DE VIDA}

Quienes padecen de $\mathrm{V}$, reportan un importante impacto negativo en la calidad de vida. Algunas encuestas determinan que el $27 \%$ considera que la $V$ causa este efecto sobre sus vidas (11). Un $42 \%$ dice no tener control en sus vidas y el $60 \%$ siente su cuerpo fuera de control. El $41 \%$ indica un impacto severo en su vida sexual (91).
En general, hay que tener en cuenta que se gastan anualmente 882 millones de dólares en EEUU, solo por dolor pélvico crónico. Sobre el 15\% pierde el trabajo y el $45 \%$ disminuye su productividad laboral debido al dolor. En estas cifras se incluye a quienes padecen vulvodinia (92). Una encuesta demostró que un cuarto de estas mujeres habían perdido trabajo en el último año debido a los síntomas de la $\mathrm{V}(91)$.

El deterioro de la calidad de vida en estas mujeres, involucra los aspectos de salud tanto mental como físico. Desde luego, mientras mayor sea el tiempo de padecimiento de esta patología, más probablemente encontraremos un estrés que llevará necesariamente al deterioro de la salud global. De esta manera es frecuente que pueda llegar a una disfunción social general, interfiriendo en el inicio o mantención de las relaciones con los pares. Emocionalmente pueden llegar a sentir frustración, tristeza e ira (4). En un estudio se demostró que tienen un deterioro mayor de la calidad de vida, al compararlas con mujeres que padecen otras patologías vulvares (4).

Los problemas sicológicos y de relación con los pares que pueden llegar a tener las mujeres con vulvodinia, no se deben solamente al dolor físico, muchas veces están influenciadas por las expectativas sobre la heterosexualidad y femineidad. Las mujeres que padecen este problema, frecuentemente se sienten como malas parejas o mujeres, ya que su dolor les puede impedir tener relaciones sexuales vaginales. Respecto a las parejas de estas mujeres, si bien algunos estudios no han demostrado que sean diferentes a otros hombres, la intensidad del dolor podría aumentar durante las relaciones sexuales, al existir hostilidad entre ellos.

Los resultados de algunas investigaciones sugieren que aún cuando los aspectos y características de las parejas de las mujeres con V, suelen ser normales, la respuesta de ellos frente a este problema, puede desempeñar un papel en la experiencia del dolor. Sin embargo, es difícil determinar qué es primero, ya que el deterioro de las relaciones sexuales por dolor, puede llevara a problemas en la pareja (88). Por otro lado, puede ser la causa de una supuesta infertilidad, que simplemente es explicada por la falta de relaciones sexuales vaginales, impedidas por la dispareunia y/o vaginismo que puede provocar la vulvodinia. Sin embargo, hay revisiones que no logran demostrar diferencias en los aspectos psicológicos al compararlas con casos controles. Esto podría ser en las primeras etapas de presentación de V. Sin embargo, con el transcurrir del tiempo, en el contexto de una patología cuyo principal síntoma y signo es el dolor de 
carácter crónico, necesariamente se asociará ansiedad y depresión.

En los estudios de V, no se ha logrado asociar a una mayor cantidad de historias de abuso sexual durante la infancia, a diferencia de aquellas que presentan sólo vaginismo. Tampoco se ha logrado asociar a una mayor presencia de base de trastornos depresivos, a diferencia de mujeres con dolor pélvico crónico, que sí parecen tenerla (93).

Otros estudios han demostrado que las mujeres con $\mathrm{V}$ pueden tener un umbral más bajo del dolor y una estimación más alta de la magnitud, además de aumento de la somatización y una imagen corporal diezmada (94). Estos últimos dos puntos, también podrían aparecer con el tiempo, producto de los temores y baja autoestima, en una mujer con dolor crónico y poca esperanza por mejorar, que ha observado a través de los años las pérdidas que esto le ha producido.

Es aceptado y reconocido que la mayoría de las mujeres con $\mathrm{V}$ tienen algún grado de afectación psicosexual. La mayoría de los médicos postulan que la permanencia del dolor crónico es el resultado de alteraciones en la inervación de la vulva y/o procesos inflamatorios, y que esto podría ser suficiente para explicar el desarrollo secundario de problemas psicosexuales. Esto constituye la teoría basada en la hipótesis neuropática. Sin embargo, un menor grupo de clínicos cree que por el contrario e inversamente, la vulvodinia puede ser el resultado de disfunciones psicosexuales pre-existentes, que debido a otros factores precipitantes, se podría expresar como dolor vulvar crónico. Esta sería en contraposición, la teoría o hipótesis somatomorfa, que involucra factores psicosociales y psicosexuales (95).

Las mujeres que padecen $\mathrm{V}$ tendrían la misma capacidad de excitación frente a situaciones que estimulan el erotismo. Sin embargo, ellas informan una significativa reducción del deseo de tener relaciones sexuales después de estímulos eróticos, seguramente por el miedo al dolor que les puede causar (96).

La mayoría de las mujeres con $\mathrm{V}$, relatan drásticos cambios en su sexualidad. El $88 \%$ reporta disminución en el interés en la actividad sexual, 94\% dice que se siente menos hábil para desarrollar su actividad sexual, y el $87 \%$ expresa que está menos dispuesto a tener relaciones sexuales. Frecuentemente se encuentra frustración en estas mujeres (97).

Sin duda, mientras más tiempo tengan $\mathrm{V}$, estas mujeres demuestran deterioro de la actividad sexual, con menores niveles de deseo, excitación y frecuencia. Con el tiempo además tienen mayor posibilidad de desarrollar ansiedad, miedo al dolor, estados de hipervigilancia, sensación de catástrofe y depresión. Todo esto a su vez tiene repercusión sobre el dolor. Esto apoya el modelo teórico que dice que la $V$ es influida entre otros por factores cognitivos y afectivos (98).

Por último debo mencionar una publicación de este año, en la que se determinó que más mujeres con vulvodinia tienen aumentos mayores de cortisol matutino, y reportan más síntomas de estrés al compararlas con mujeres sanas de la misma edad (99). Esto es producto del mismo estrés que les provoca este dolor y lo que significa o se relaciona con él.

No puedo dejar de mencionar, que algunas revisiones han demostrado que las mujeres que tienen $\mathrm{V}$, tienen mayor probabilidad de haber sufrido abuso en la etapa infantil, ya sea de tipo físico o sexual. Sin embargo, la gran mayoría de las publicaciones, no logran encontrar esta diferencia al compararla con poblaciones control, que no padecen V (100).

\section{CONCLUSIONES}

La vulvodinia constituye una patología frecuentemente no diagnosticada, a la cual equivocadamente, al no encontrar una posible explicación lógica, se atribuye exclusivamente a factores psicológicos o alteraciones emocionales. Es poco lo que conocemos respecto a su fisiopatología, sin embargo, ya se han identificado algunas alteraciones como en citoquinas, que podrían explicar la presencia de zonas de hiperalgesia. En un tejido con posibles alteraciones en la inervación y con cambios a nivel de los nociceptores. Todo esto constituye la hipótesis neuropática. La vulvodinia es una patología multifactorial y de alta complejidad.

Los aspectos psicológicos, juegan un rol muy importante en la génesis de las alteraciones psicosexuales secundarias, que presentan estas mujeres, por lo que muchas veces constituyen una parte importante en la terapia, reafirmando la hipótesis psicosomática en la etiopatogenia de estas alteraciones.

Todos los esfuerzos por comprender mejor las bases fisiopatológicas de esta enfermedad crónica, permitirán buscar nuevas terapias, que se agregarán a las ya conocidas, como modificación de la dieta, fármacos, kinesiterapia, psicoterapia y cirugía.

Los ginecólogos debemos estar atentos a la aparición en mayor frecuencia de mujeres que se atreven a consultar por esta causa, y se debe derivar a aquellos que tienen la experiencia en su tratamiento, ya que por tratarse de una patología cuyo síntoma principal es el dolor, requiere un 
seguimiento especial y un soporte de un equipo de trabajo. En su diagnóstico, se deben descartar patologías diferenciales, tales como fenómenos infecciosos o inflamatorios.

Los tratamientos deben incluir los diferentes aspectos, desde la terapia farmacológica sistémica y local, la kinesiterapia y la psicoterapia. La cirugía debe realizarse en quienes no responden con las terapias tradicionales.

\section{BIBLIOGRAFÍA}

1. ACOG Committee on Gynecologic Practice. ACOG Committee Opinion: Number 345, October 2006: Vulvodynia. Obstet Gynecol 2006;108(4):1049-52.

2. Hallado en: http://www.icd9data.com/2009/Volume1/580-629/617-629/625/default.htm

3. Boardmen LA, Cooper AS, Blais LR, Raker CA. Topical gabapentin in the treatment of localized and generalized vulvodynia. Obstet Gynecol 2008;112(3):57985.

4. Ponte M, Klemperer E, Sahay A, Chren MM. Effects of vulvodynia on quality of life. J Am Acad Dermatol 2009;60(1):70-6.

5. Haefner HK, Collins ME, Davis GD, Edwards L, Foster DC, Hartmann EH, et al. The vulvodynia guidline. J Low Genit Tract Dis 2005;9(1):40-51.

6. Skene AJ. Treatise on the diseases of women, for the use of students and practitioners. New York: Appleton \& Company, 1889.

7. Thomas TG, Munde PF: Hyperaesthesia of vulva. The Diseases of Women. Philadelphia:Lea Brothers \& Co.1891.

8. Kelly HA: Gynecology. New York: Appleton \& Company.1928.

9. Marin MG, King R, Sfameni S, Dennerstein G. Adverse behavioral and sexual factors in chronic vulvar disease. Am J Obstet Gynecol 2000;183(1):34-8.

10. Tuma R, Bornstein J. Vulvar pain syndrome (vulvodynia)--dilemmas in terminology. Harefuah 2006;145(3):215-8.

11. Arnold LD, Bachmann GA, Rosen R, Rhoads GG. Assessment of vulvodynia symptoms in a sample of US women: a prevalence survey with a nested case control study. Am J Obstet Gynecol 2007;196(2):128.

12. Reed BD, Haefner HK, Sen A, Gorenflo DW. Vulvodynia incidence and remission rates among adult women: a 2-year follow-up study. Obstet Gynecol 2008;112(2 Pt 1):231-7.

13. Harlow BL, Stewart EG. A population-based assessment of chronic unexplained vulvar pain: have we underestimated the prevalence of vulvodynia?. J Am Med Womens Assoc 2003;58(2):82-8.

14. Resident Population Estimates of the United States by Age and Sex. April1, 1990-July 1,1999. Washington DC: Population Estimates Program, Population Division, US Census Bureau; 2000.

15. Goetsch MF. Vulvar vestibulitis: Prevalence and historic features in a general gynecologic practice population. Am J Obstet Gynecol 1991;164(6 Pt 1):1609-14.
16. Reed BD, Cantor LE. Vulvodynia in preadolescent girls. J Low Genit Tract Dis 2008;12(4):257-61.

17. Lavy RJ, Hynan LS, Haley RW. Prevalence of vulvar pain in an urban, minority population. J Reprod Med 2007;52(1):59-62.

18. Foster DC. Vulvar disease. Obstet Gynecol 2002;100(1):145-63.

19. Smart OC, MacLean AB. Vulvodynia. Curr Opin Obstet Gynecol 2003;15(6):497-500.

20. Gerber S, Bongiovanni AM, Ledger WJ, Witkin SS. Defective regulation of the proinflammatory immune response in women with vulvar vestibulitis syndrome. Am J Obstet Gynecol 2002;186(4):696-700.

21. Foster DC, Piekarz KH, Murant TI, LaPoint R, Haidaris CG, Philipps RP. Enhanced synthesis of proinflammatory cytokines by vulvar vestibular fibroblasts: implications for vulvar vestibulitis. Am J Obstet Gynecol 2007;196(4):346.e1-8.

22. Foster DC, Sazenski TM, Stodgell CJ. Impact of genetic variation in interleukin-1 receptor antagonist and melanocortin-1 receptor genes on vulvar vestibulitis syndrome. J Reprod Med 2004;49(7):503-9.

23. Halperin R, Zehavi S, Vaknin Z, Ben-Ami I, Pansky M, Schneider D. The major histopathologic characteristics in the vulvar vestibulitis syndrome. Gynecol Obstet Invest 2005;59(2):75-9.

24. Bohm-Starke N, Hilliges M, Falconer C, Rylander E. Neurochemical characterization of the vestibular nerves in women with vulvar vestibulitis syndrome. Gynecol Obstet Invest 1999;48(4):270-5.

25. Tympanidis $P$, Casula MA, Yiangou Y, Terenghi G, Dowd $P$, Anand $P$. Increased vanilloid receptor VR1 innervation in vulvodynia. Eur Pain 2004;8(2):129-33.

26. Matthews PJ, Aziz Q, Facer P, Davis JB, Thompson DG, Anand P. Increased capsaicin receptor TRPV1 nerve fibres in the inflamed human oesophagus. Eur $\mathrm{J}$ Gastroenterol Hepatol 2004;16(9):897-902.

27. Bohm-Starke N, Hilliges M, Brodda-Jansen G, Rylander E, Torebjörk E. Psychophysical evidence of nociceptor sensitization in vulvar vestibulitis syndrome. Pain 2001;94(2):177-83.

28. Eide PK. Wind-up and the NMDA receptor complex from a clinical perpective. Eur J Pain 2000;4(1):5-15.

29. Bohm-Starke N, Hilliges M, Blomgren B, Falconer C, Rylander $\mathrm{E}$. Increased blood flow and erythema in posterior vestibular mucosa in vulvar vestibulitis. Obstet Gynecol 2001;98(6):1067-74.

30. Ashman RB, Papadimitriou JM, Ott AK, Warmington JR. Antigens and immune response in Candida albicans infection.Immunol Cell Biol 1990;68(Pt 1):1-13.

31. Franklyn KM, Warmington JR, Ott AK, Ashman RB. An immunodominant antigen of Candida albicans shows homology to the enzyme enolase. Immunol Cell Biol 1990;68(Pt 3):173-178.

32. Marinoff $S$, Turner M. Hypersensivity of vaginal candidiasis or treatment vehicles in the pathogenesis of minor vestibular gland syndrome. J Reprod 1986;31(9):796-799.

33. Foster DC, Dworkin RH, Wood RW. Effects of intradermal foot and forearm capsaicin injection in normal and vulvodynia-afflicted women. Pain 2005;117(12):128-36. 
34. Giesecke J, Reed BD, Haefner HK, Giesecke T, Clauw DJ, Gracely RH. Quantitative sensory testing in vulvodynia patients and increased peripheral pressure pain sensitivity. Obstet Gynecol 2004;104(1):126-33.

35. Pukall CF, Binik YM, Khalife S, Amsel R, Abbott FV. Vestibular tactile and pain thresholds in women with vulvar vestibulitis syndrome. Pain 2002;96(1-2):16375.

36. Granot M. Personality traits associated with perception of noxious stimuli in women with vulvar vestibulitis syndrome. J Pain 2005;6(3):168-73.

37. Foster DC, Robinson JC, Davis KM. Urethral pressure variation in women with vulvar vestibulitis syndrome. Am J Obstet Gynecol 1993;169(1):107-12.

38. Bornstein J, Shapiro S, Rahat M, Goldshmid N, Goldik $Z$, Abramovic, et al. Polymerase chain reaction search for viral etiology of vulvar vestibulitis syndrome. Am J Obstet Gynecol 1996;175(1):139-44.

39. Morin C, Bouchard C, Brisson J, Fortier M, Blanchette $C$, Meisels $A$. Human papillomaviruses and vulvar vestibulitis. Obstet Gynecol 2000;95(5):683-7.

40. Gaunt G, Good AE, McGovern RM, Stanhope CR, Gostout BS. Human papillomavirus in vulvar vestibulitis syndrome. J Reprod Med 2007;52(6):485-9.

41. Oates JK.. Focal vulvitis and localised dyspareunia. Genitourin Med 1990;66(1):28-30.

42. Damsted-Petersen C, Boyer SC, Pukall CF. Current perspectives in vulvodynia. Womens Health (Lond Engl) 2009;5(4):423-436.

43. Nguyen RH, Swanson D, Harlow BL. Urogenital infections in relation to the occurrence of vulvodynia. $J$ Reprod Med 2009;54(6):385-92.

44. Smith EM, Ritchie JM, Galask R, Pugh EE, Jia J, Ricks-McGillan J. Case-control study of vulvar vestibulitis risk associated with genital infections. Infect Dis Obstet Gynecol 2002;10(4):193-202.

45. Oaklander AL, Rissmiller JG. Postherpetic neuralgia after shingles: an under-recognized cause of chronic vulvar pain. Obstet Gynecol 2002;99(4):625-8.

46. Ragozzino MW, Melton LJ, Kurland LT, Chu CP, Perry HO. Population-based study of herpes zoster and its sequelae. Medicine (Baltimore) 1982;61(5):310-316.

47. Gardella B, Porru D, Ferdeghini F, Martinotti Gabelleotti E, Nappi RE, Rovereto B, et al. Insight into urogynecologic features of women with instertitial cistitis/ painful bladder. Eur Urol 2008;54(5):1145-51

48. Carrico DJ, Sherer KL, Peters KM. The relationship of interstitial cystitis/painful bladder syndrome to vulvodynia. Urol Nurs 2009;29(4):233-8.

49. Danielsson I, Sjoberg I, Wikman M. Vulvar vestibulitis: medical, pschosexual and psychosocial aspects, a case-control study. Acta Obstet Gynecol Scand 2000;79(10):872-878.

50. Engman M, Wijma K, Wijma B. Itch and burning pain in women with partial vaginismus with or without vulvar vestibulitis. J Sex Marital Ther 2007;33(2):171-86.

51. Goetsch MF. Surgery combined with muscle therapy for dyspareunia from vestibulitis: an observational study. J Reprod Med 2007;52(7):597-603.

52. Ter Kuile MM, Van Lankveld JJ, Vlieland CV, Willekes C, Weijenborg PT. Vulvar vestibulitis syndrome: an important factor in the evaluation of lifelong vaginis- mus? J Psychosom Obstet Gynaecol 2005;26(4):2459.

53. Raiteri R, Sinicco A, Pippione M, Tomasini C, Solaroli C, Vitale L, et al. Vulvodynia and HIV: causal or casual association? Genitourin Med 1997;73(6):545-7.

54. Reed BD, Gorenflo DW, Haefner HK. Genralized vulvar dysesthesia vs. vestibulodynia. Are they distinct diagnoses?. J Reprod Med 2003;48(11):858-6.

55. Haefner HK. Report of the International Society for the Study of Vulvovaginal Disease terminology and classification of vulvodynia. J Low Genit Tract Dis 2007;11(1):48-9.

56. Arnold LD, Bachmann GA, Rosen R, Kelly S, Rhoads GG. Vulvodynia: characteristics and associations with comorbidities and quality of life. Obstet Gynecol 2006;107(3):617-24.

57. Lema R, Ricci P. Atrapamiento del Nervio Pudendo: un Síndrome por conocer. Rev Obstet Ginecol 2006;71(3):207-215.

58. Reed BD, Haefner HK, Harlow SD, Gorenflo DW, Sen A. Reliability and validity of self-reported symptoms for predicting vulvodynia. Obstet Gynecol 2006;108(4):906-913.

59. Reed BD, Crawford S, Couper M, Cave C, Haefner HK. Pain at the vulvar vestibule: A web-based survey. J Low Genit Tract Dis 2004;8(1):48-57.

60. Friedrich EG Jr. Vulvar vestibulitis síndrome. J Reprod Med 1987;32(2):110-4

61. Peckham B, Maki D, Patterson J, Gholan-Reza H. Focal vulvitis: A characteristic syndrome and cause of dyspareunia. Features, natural history, and management. Am J Obstet Gynecol 1986;154(4):855-864.

62. Omoiqui $S$. The biochemical origin of pain: the origin of all pain is inflammation and the inflammatory response. Part 2 of 3 - inflammatory profile of pain syndrome. Med Hypotheses 2007;69(6):1169-78.

63. Reed BD, Caron AM, Gorenflo DW, Haefner HK. Treatment of vulvodynia with tricyclic antidepressants: efficacy and associated factors. J Low Genit Tract Dis 2006;10(4):245-51.

64. Harris G, Horowitz B, Borquida A. Evaluation of gabapentin in the treatment of generalized vulvodynia, unprovoked. J Reprod Med 2007;52(2):103-6.

65. Zolnoun DA, Hartmann KE, Steege JF. Overnight 5\% lidocaine ointment for for treatment of vulvar vestibulitis. Obstet Gynecol 2003;102(1):84-7.

66. McCleane G. Topical application of doxepin hydrochloride, capsaicin and a combination of both produces analgesia in chronic human neuropathic pain: a randomized, double-blind, placebo-controlled study. J Clin Pharmacol 2000;49(6):574-9.

67. Nyirjesy P, Sobel JD, Weitz MV, Leaman DJ, Small MJ, Gelone SP. Cromolyn cream for recalcitrant idiopathic vulvar vestibulitis: results of a placebo controlled study. Sex Transm Infect 2001;77(1):53-7.

68. Walsh KE, Berman JR, Berman LA, Vierregger K. Safety and efficacy of topical nitroglycerin for treatment of vulvar pain in women with vulvodynia: a pilot study. J Gend Specif Med 2002;5(4):21-7.

69. Steinberg AC, Oyama IA, Rejba AE, Kellogg-Spadt S, Whitmore KE. Capsaicin for the treatment of vulvar vestibulitis. Am J Obstet Gynecol 2005;192(5):1549-53. 
70. Murina F, Tassan P, Roberti P, Bianco V. Treatment of vulvar vestibulitis with submucous infiltrations of methylprednisolone and lidocaine. An alternative approach. J Reprod Med 2001;46(8):713-6.

71. Dede M, Yenen MC, Yilmaz A, Baser I. Successful treatment of persistent vulvodynia with submucous infiltration of betamethasone and lidocaine. Eur J Obstet Gynecol Reprod Biol 2006;124(2):258-9.

72. McKay E, Kaufman RH, Doctor U, Berkova Z, Glazer $\mathrm{H}$, Redko V. Treating vulvar vestibulitis with electromyographic biofeedback of pelvic floor musculature. $\mathrm{J}$ Reprod Med 2001;46(4):337-42.

73. Dionisi B, Anglana F, Inghirami P, Lippa P, Senatori $R$. Use of transcutaneous electrical stimulation and biofeedback for the treatment of vulvodynia (vulvar vestibular syndrome):result of 3 years of experience. Minerva Ginecol 2008;60(6):485-91.

74. Dionisi B, Anglana F, Inghirami P, Lippa P, Senatori $R$. Use of transcutaneous electrical stimulation and biofeedback for the treatment of vulvodynia (vulvar vestibular síndrome):result of 3 years of experience. Minerva Ginecol 2008;60(6):485-91.

75. Reissing ED, Brown C, Lord MJ, Binik YM, Khalifé $\mathrm{S}$. Pelvic floor muscle functioning women with vulvar vestibulitis syndrome. J Psychosom Obstet Gynaecol 2005;26(2):107-113

76. Powell J, Wojnarowska F. Acupunture for vulvodynia. J R Soc Med 1999;92(11):579-81.

77. Pukall C, Kandyba K, Amsel R, Khalifé S, Binik Y. Effectiveness of hypnosis for the treatment of vulvar vestibulitis syndrome: a preliminary investigation. J Sex Med 2007;4(2):417-25.

78. Lavy Y, Lev-Sagie A, Hamani Y, Zacut D, Ben-Chetrit A. Modified vulvar vestibulectomy: simple and effective surgery for the treatment of vulvar vestibulitis. Eur $\mathrm{J}$ Obstet Gynecol Reprod Biol 2005;120(1):91-5.

79. Leclair CM, Goetsch MF, Lee KK, Jensen JT. KTPnd:YAG laser therapy for the treatment of vestibulodynia: a follow-up study. J Reprod Med 2007;52(1):53-8.

80. Bornstein J, Zarfati D, Cohen C, Tendler R, Ophir E. Vestibulodynia--the Israeli experience. Harefuah 2009;148(7):465-70.

81. Goetsch MF. Patients' assessments of a superficial modified vestibulectomy for vestibulodynia. J Reprod Med.2008;53(6):407-412.

82. Eva LJ, Narain S, Orakwue CO, Luesley DM. Is modified vestibulectomy for localized provoked vulvodynia an effective long-term treatment? Afollow-up study. J Reprod Med 2008;53(6):435-40.

83. Solomons CC, Melmed MH, Heitler SM. Calcium citrate for vulvar vestibulitis. Acase report. J Reprod Med 1991;36(12):879-82.

84. Nyirjesy $P$, Halpern M. Medical management of vulvar vestibulitis:results of a sequential treatment plan. Infect Dis Obstet Gynecol 1995;3(5):193-7.

85. Poole S, Ravenhill G, Munday PE. A pilot study of the use of low oxalate diet in the treatment of vulval vestibulitis. J Obstet Gynaecol 1999;19(3):271-2.

86. Bergeron S, Binik YM, Khalifé S, Pagidas K, Glazer HI, Meana $\mathrm{M}$, et al. A randomized comparison of group cognitive--behavioral therapy, surface electromyographic biofeedback, and vestibulectomy in the treatment of dyspareunia resulting from vulvar vestibulitis. Pain 2001;91(3):297-306.

87. Sutton KS, Pukall CF, Chamberlain S. Pain ratings, sensory thresholds, and psychosocial functioning in women with provoked vestibulodynia. J Sex Marital Ther 2009;35(4):262-81.

88. Jodoin M, Berqeron S, Khalifé S, Dupuis MJ, Desrochers G, Lecler B. Male partners of women with provoked vestibulodynia: attributions for pain and their implications for dyadic adjustment, sexual satisfaction, and psychological distress. J Sex Med 2008;5(12):2862-70.

89. Cecilio SB, Zaghi S, Cecilio LB, Correa CF, Fregni F. Exploring a novel therapeutic approach with noninvasive cortical stimulation for vulvodynia. Am J Obstet Gynecol 2008;199(6):e6-7.

90. Bergeron S, Khalifé S, Glazer HI, Binik YM. Surgical and behavioral treatments for vestibulodynia: twoand-one-half year follow-up and predictors of outcome. Obstet Gynecol 2008;111(1):159-66.

91. Arnold LD, Bachmann GA, Rosen R, Kelly S, Rhoads GG. Vulvodynia: characteristics and associations with comorbidities and quality of life. Obstet Gynecol 2006;107(3):617-24.

92. Mathias SD, Kuppermann M, Liberman RF, Lipschutz RC, Steege JF. Chronic pelvic pain: prevalence, health-related quality of life, and economic correlates. Obstet Gynecol 1996;87(3):321-327.

93. Reed BD, Haefner HK, Punch MR, Roth RS, Gorenflo DW, Gillespie BW. Psychosocial and sexual functioning in women with vulvodynia and chronic pelvic pain. A comparative evaluation. J Reprod Med 2000;45(8):624-32.

94. Granot M, Lavee Y. Psychological factors associated with perception of experimental pain in vulvar vestibulitis síndrome. J Sex Marital Ther 2005;31(4):285-302.

95. Lynch PJ. Vulvodynia as a somatoform disorder. J Reprod Med 2008;53(6):390-6.

96. Payne KA, Binik YM, Pukall CF, Thaler L, Amsel R, Khalifé $S$. Effects of sexual arousal on genital and non-genital sensation: a comparison of women with vulvar vestibulitis syndrome and healthy controls. Arch Sex Behav 2007;36(2):289-300.

97. Sackett S, Gates E, Heckman-Stones C, Kobus AM, Galask R. Psycholsexual aspects of vulvar vestibulitis. J Reprod Med 2001;46(6):593-8.

98. Desrochers G, Bergerson S, Khalifé S, Dupuis MJ, Jodoin M. Fera avoidance and self-efficacy in relation to pain and sexual impairtment in women with provoked vestibulodynia. Clin J Pain.2009;25(6):520-7.

99. Ehrström S, Kornfeld D, Rylander E, Bohm-Starke N. Chronic stress in women with localized provoked vulvodynia. J Psychosom Obstet Gynaecol 2009;30(1):73-79.

100.Dalton VK, Haefner HK, Reed BD, Senapati S, Cook A. Victimization in patients with vulvar dysesthesia/ vestibulodynia. Is there an increased prevalence? J Reprod Med 2002;47(10):829-34. 